\title{
HispaSim: a Web Application for Satellite Link Budget Optimization and Management
}

\author{
Luis Escolar Haro ，Gregorio Juliana Quirós ，Álvaro Rodríguez Villalba , Ramón Martínez Rodríguez-Osorio \\ Pablo Honold , Antonio Arana , Alejandro Martínez , Inés Sanz
}

\begin{abstract}
Adequate link budget tools are required by satellite operators to generate optimized transmission plans that satisfies customer diverse demands using transponder resources (power and bandwidth) efficiently. Satellite operator has to face with a number of communication scenarios depending on the service. These scenarios cover from forward links used for DTH (Directto-Home) to bidirectional networks where both forward and returns can share the transponder resources. Moreover, a transition from desktop implementations to web-based applications is a must for satellite link budget tools. In this contribution, we present a web-based application entitled HispaSim conceived for the analysis and optimization of satellite link budgets that incorporates administration of users and database. HispaSim application has been developed by the Grupo de Radiación of ETSIT-UPM (Escuela Técnica Superior de Ingenieros de Telecomunicación, Universidad Politécnica de Madrid) developed for Hispasat and GMV.
\end{abstract}

Keywords-satellite link budget; web application; link budget optimization; DVB-S2; DVB-S2x; satellite network; satellite communication services.

\section{INTRODUCTION}

The massive demand for communication satellite services is increasing and satellite operators must satisfy user demands in terms of availability and quality of service.

Adequate link budget tools are required by the operator to generate optimized transmission plans. Satellite operator has to face with a number of communication scenarios depending on the service. These scenarios cover from forward links used for DTH (Direct-to-Home) to bidirectional networks where both forward and returns can share the transponder resources. In addition, satellite operators often deal with customers that require different needs for their communication services and networks.

The efficient use of the satellite transponder resources in terms of power and bandwidth leads to the optimization of the throughput for a specified availability.

With the advent of DVB-S2 [1] and DVB-S2x [2], link budget calculations must deal with dynamic and adaptive technologies such as VCM (Variable Coding and Modulation) and ACM (Adaptive Coding and Modulation) that must be considered during the simulation, as well as conventional CCM operation (Constant Coding and Modulation). Moreover, these tools should be aimed not only for the link budget calculations but also offer the SatCom engineers with a set of results coming that can be presented to the customer in straight- forward format. Thus, advanced tools for the management of satellite link budgets must be designed and implemented.

In contrast to desktop applications, a web-platform accessible from conventional Internet browsers offers a number of significant advantages: no installation or downloads are required in personal computers or laptops (leading to an easier maintenance), updates are implemented in the web server, they are independent of the operating system, and. In the recent years, a trend to substitute desktop programs by web-based applications has been consolidated in the ICT industry. The main remark to web based applications is the need to have an Internet connection available at any time.

In this contribution, we present a web-based application entitled HispaSim conceived for the analysis and optimization of satellite link budgets that incorporates administration of users and database. HispaSim application has been designed and implemented by the Grupo de Radiación of ETSIT-UPM (Escuela Técnica Superior de Ingenieros de Telecomunicación, Universidad Politécnica de Madrid) developed for Hispasat and GMV.

This paper is organized as follows. Section II presents the requirements of HispaSim and section III details the architecture of the application. Section IV describes the core part of HispaSim, namely, the link budget tool. Section V shows the simulation flow including the process to exchange information in the process of constructing a simulation scenario. In section VI example case studies implemented in the application are presented with their corresponding results, and finally section VII draws the main conclusions and future evolution of HispaSim.

\section{APPLICATION REQUIREMENTS}

An extensive list of requirements defines the framework of HispaSim from the user to the functionalities to the security, maintenance and administration of the application. A summary of the most significant features of HispaSim are presented below:

- Analysis and optimization of satellite link budgets using different criteria

- Simulation of satellite link budgets in forward, return and networks scenarios

- Organization of link budget analysis in projects and simulations in self-contained files

- Representation of link budget results in contour plots using a web map service 
- Support of diverse geometries (point, multipoint and areas) and a database of cities and countries

- $\quad$ Storage of simulation results and reports

- Application to register and validate a ground station

- Management of a database including modem technologies, ground stations, coordination agreements and satellite transponders

- $\quad$ Processing of antenna pattern files specified in different formats

- Modification of satellite and antenna attitude angles (roll, pitch, yaw) to evaluate its impact on link budget performance

- Consideration of coordination agreements in uplink and downlink, as well as emission limits

- Web-based application with an easy-to-use user interface that can be launched from most extended Internet browsers

- $\quad$ Reporting of link budget results in user-defined templates

- Administration of user groups and profiles (permissions)

In order to fulfil with the proposed requirements, HispaSim is conceived as a web application formed by a group of tools accessible form the main window. The tools that form HispaSim are:

- $\quad$ Link Budget: tool for the analysis and design of satellite link budgets.

- Database Manager: application for the management of database objects, with access to the edition of HispaSim objects as satellites, transponders, stations, etc.

- Administration: application for the management of users, groups, profiles, report templates, and critical information of HispaSim.

- Ground stations: application used to request the validation of a station to be incorporated in the HispaSim database objects.

Depending on the user groups and profiles, each user has access to all or a particular group of tools. HispaSim administrator is responsible for the user management, whose profile and group can be changed according to user demands. While user groups define a set of objects accessible by the users in the group, the profiles define the permissions or capabilities to use the functionalities of the application.

\section{HISPASIM ARCHITECTURE}

\section{A. Architecture}

The application is organized in several interconnected modules that provide different services as shown in the block diagram of Fig. 1. They are described next, starting from the most external layer.

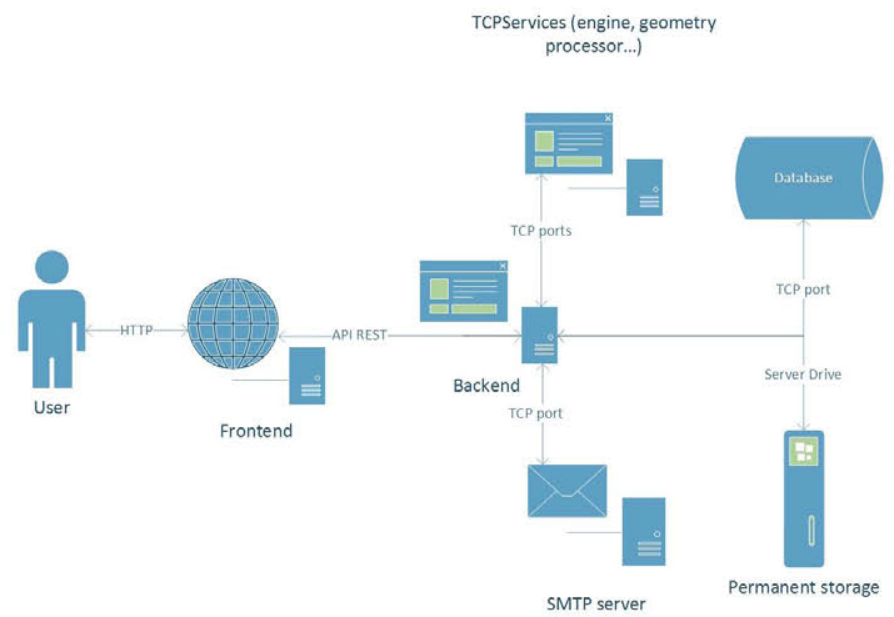

Fig. 1. HispaSim architecture.

The part of the web application defined as frontend is the one executed in the browser of the user's machine. Since a simulator requires heavy interaction with the user performing small configuration changes in the simulations, a frontend based on a multi-page architecture would imply a significant amount of complete page reloads, requiring more HTTP requests and more HTML renders server-side, since the backend would be in charge of filling the HTML with content. By choosing a one-page application architecture, there is only one complete page load each time the user accesses the webpage, reducing the total load on the server. Also, since the one-page application frameworks provide client-side page routing, the load of partial pages is faster and more lightweight.

The main disadvantages of single-page applications are the memory footprint of the document and the latency of the initial download of the elements. To avoid them and modularize the frontend we separated it in four sub applications, related to the role of the user that will use each one:

- $\quad$ Link Budget: This application is meant to be used by engineers and clients to make simulations.

- Database manager: This application is meant to be used by engineers to modify the database elements.

- Ground stations: This application is meant to be used by clients to create and administer their ground stations.

- Administration: This application is meant to be used by the administrator to create and modify sensible data.

These four sub applications are accessed through a portal. The client-side routing is divides each application in multiple partial views, each one with one HTML partial and one Javascript controller, but allowing nested partials and controllers. Each controller is in charge of the following tasks:

- Data retrieval: Each controller retrieves its own data from the server using the HTTP REST API that it provides.

- Data presentation: Each controller parses and preprocesses the data retrieved and displays it to the user.

- Data update: Each controller receives the user's input from his/her data scope and performs any operations needed to persist it in the system. 
This modular design allows for a heavy code reutilization, since small controllers and HTML partials are fully independent and can be used multiple times across the app.

Behind the scenes lies the backend, whose design is focused on delivering the best performance, exploiting the advantages of different technologies and communicating them in order to ensure that their strengths complement. The interface with the frontend is an HTTP REST API as stated above, powered by a Node.JS server. This interface is completely agnostic to the services that consume it, allowing a great deal of flexibility on that end.

The server manages the insertion, deletion and update of data from and to different storage systems (mainly on a filesystem and a MySQL database), and exposes it in the form of JSON objects. This consistent interface abstracts the storage, so the underlying technologies can be replicated, enhanced or replaced depending on the requirements of a setup.

On the other hand, it connects to a series of Java TCP servers (TCP services from now on) that wrap the tools that are more CPU intensive. The list of tools is the following:

- Link budget simulator

- Antenna file processor

- Geometry utilities (geojson merger, simplifier...)

\section{- Simulation report generator}

This design offers several advantages, as the calculations that the system has to perform can be very demanding on the hardware and Node.JS (while a perfect fit for the REST API provider) is not the optimal technology for this kind of tasks. This way we can ensure the interface will be responsive to multiple users while the system is under heavy load, and unrelated calls (user administration tasks, for example) won't suffer from delays if a heavy simulation or antenna file processing is taking place

Thus, while the web backend is focused on serving multiple users in a timely, non-blocking fashion; the TCP services feature a FIFO queue for the different jobs that users schedule. Since these jobs can potentially take up a great amount of resources (mostly RAM and CPU time), they must be arranged this way so multiple concurrent users won't collapse the system. The trade-off of resource bounding vs. execution time can be softened as the TCP services could be replicated (for a multi-core machine) or even set up on different machines.

Other auxiliary services (SMTP email server, HTTPS proxy) interface with the Node.JS server via different plugins. The design allows for the replication of any and every component listed, so the scalability of the system is taken into account.

All encryption and authentication procedures are delegated to an external proxy in order to maintain modularity. As different clients require different setups and may have authentication backends already in place, our system reads certain HTTP headers in order to differentiate and authorize users. We have successfully tested it with Nginx, Apache and a custom Node.JS proxy.

\section{B. Technologies}

To provide the services specified in the previous point, the following technologies were used:
AngularJS was chosen as the frontend framework as it is a powerful and reliable technology oriented to single-page applications, easily integrable with other frontend technologies such as bootstrap and JQuery-based libraries. It provides clientside routing, hierarchical data scope and controller system, and two-way data binding between the views and the controllers, giving us the perfect tools for building a highly modular app like the simulator.

CesiumJS has been chosen as the map framework because of its open-source license, configurability and simplicity. We designed a custom AngularJS controller wrapper around it, providing us with a simple way of displaying geographical data in the simulator.

Bootstrap was selected as the CSS framework as it is a simple and well tested library that allows to create a responsive web application using a simple grid system. It also provides a set of basic UI elements that are frequently used across the app.

Node.JS was chosen as the server framework as it is a stateof-the-art technology while remaining stable and reliable enough for a production system. Its event-driven programming model based on a single-threaded event loop allows us to exert precise control on how the resources of the server are spent, while allowing us to serve a high amount of concurrent users. It can be scaled to take up as many resources as needed on a multi-core machine or a multiple server setup thanks to its clustering capabilities, and its ever-growing package manager environment (NPM) gives us a lot of tools to extend the functionality of the system. Besides, using the same language as our preferred frontend (JavaScript) really sped up the development, and using JSON is a natural fit for a HTTP REST API.

On the other hand it has a highly consistent multiplatform API. Setting it up on different operative systems is quite simple, as it is not affected by common pitfalls like filesystemdependent routes or custom scripts/executables.

As stated before, the CPU intensive tasks are offloaded to several utilities written in Java. We designed a custom, very thin TCP server wrapper around these tools, as a solution like Tomcat was excessive for our needs.

A SQL database was chosen due to its popularity and different implementations available. Also, the maturity of the ORMs available for Node.JS conditioned this decision, as the models and migrations could be defined on the code itself, abstracting them from the underlying database technology.

Finally, a combination of Gulp.JS and several of its plugins are use together with Maven (for the Java code) in order to setup an automatic build pipeline. It supports several configurations and tasks, but its most important automation capabilities are:

\section{- $\quad$ Project structure check.}

- Compilation and minimization of the frontend scripts.

- Automatic code testing.

- Compilation of the Java code and dependencies into a single .jar file.

- Creation of default configuration files.

- Setup of the default data and templates. 
- Automatic build packaging into a zip file with timestamps.

These tools also allow the easy setup and deployment of the system into a continuous integration/deployment server (using Jenkins, Docker and/or other mechanisms).

\section{HisPaSim LiNK BUDGET APPLICATION}

The link budget application tool is the core part of the application as it interacts with the rest of applications (user management, database). 0 shows the architecture of the link budget application.

To create a link budget analysis the following entities must be considered:

- $\quad$ Project: entity that includes a number of simulations and associated reports.

- Simulation: entity that defines a scenario for the realization of a link budget analysis that includes a satellite and transponder, one or several services, composed by a set of stations (hub and remotes) with their associated locations and carriers. Users may generate more than one report for each simulation. One project contains several simulations, and each simulation may use a different satellite and/or transponder.

Link budget contains the following modules which are used by the user to configure the simulation and manage the existing projects:

- Project selector: it shows in a sortable table or grid the existing projects. Projects can be deleted, created and duplicated.

- Project editor: it shows the contents of the project and he user can edit different parameters. It is divided in three parts: 1) form with project information, 2) set of simulations included in the project, and 3) reports with simulation results generated by the user. Simulations can be duplicated (using a different name) and deleted, and reports can downloaded and deleted.

- Simulator: it is divided in two parts:

- Scenario: information about the simulation and launch of the simulator. It includes the definition of general parameters of the simulation, selection of the satellite, transponder and configuration of its operation point, interferences, and service.

- Results: divided into three menus, namely Forward, Return and Network.

Regarding interferences, several types can be defined during the configuration of the simulation:

- Internal interferences: due to operation of satellites and stations operating in the same orbital positions.

- External interferences: operation of station and satellites in neighbor orbital positions.

- Coordination agreements with other satellite operators: they can be defined in the simulation or be read from the database.

- $\quad$ Out of band emissions (only in uplink).

- $\quad$ Other C/I (only in downlink).
- Cochannel crosspolar interference: due to misalignment of polarization axis and XPD of antennas antennas operating in same channel but using an orthogonal polarization.

The service is configured through the selection of a hub, remotes and carrier.

Regarding EIRP mode, the user can select the method to calculate the EIRP transmitted by the ground stations. Common modes in forward and return scenarios are:

- Minimum: engine calculates the minimum required EIRP to solve the link budget with the specified availability.

- Nominal: user introduces the EIRP value in the hub or remotes that will be used by the engine.

- Balanced: engine calculates the required EIRP in the hub or remotes so power and bandwidth consumptions in the transponder are balanced.

- Maximum: engine uses the maximum EIRP of the ground station to solve the link budget.

The configuration of the modem technology implies the import of modem technology, selection of MODCODs and operation (ACM, VCM, CCM, Multi CCM).

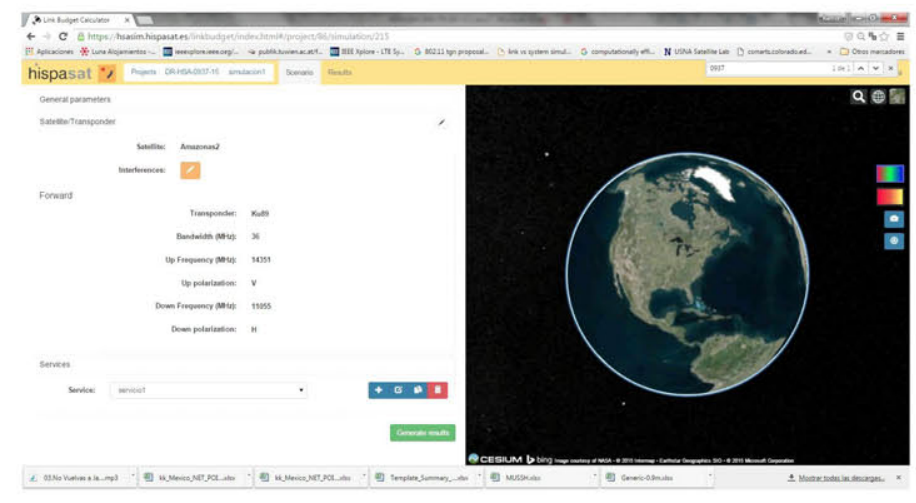

Fig. 2. User interface of the link budget tool.

\section{Simulation FlowChaRT}

In the link budget, the configuration of a simulation involves the participation of the four modules of the application: frontend, backend, engine and database. The creation of the scenario is based on the exchange of information between the modules to populate different JSON files. The use of a lightweight and flexible format like JSON (JavaScript Object Notation) to interchange data is especially suited for web applications working with Javascript [4]. The interaction of the user with the interface involves a communication between front-end and back-end to fill a JSON file denoted as simFile.json. This file contains all the information needed by the engine to calculate the link budget.

The JSON files are populated either with data introduced directly by the user through the interface, or with data obtained from the database. The former is the case of satellites, transponders, ground stations or locations. The latter is the case of parameters of the simulation such as the EIRP mode of the ground stations, availability, or the operation of the modem technology. Once the configuration of the scenario is completed, the user launches the simulation and the simFile.json file is processed by the engine to carry out the link budget analysis and optimization. 
Link budget results are stored in another JSON file called resultFile.json. This file contains information of the simulation results following a predefined structure that is interpreted by the front-end to present the requested information in the result screen. When the user selects a result variable to visualize the contour in the map, the front-end request the contours to the back-end. These contour results are generated by the engine and displayed in the map in the results screen. An intermediate JSON file is generated by the engine with a specific structure containing contour results or prepared for the generation of a report under a specified template.

An authorization and access layer has been incorporated. It allows that user has access exclusively to the objects of the database that correspond to his/her user group, and to avoid the access of unauthorized users.

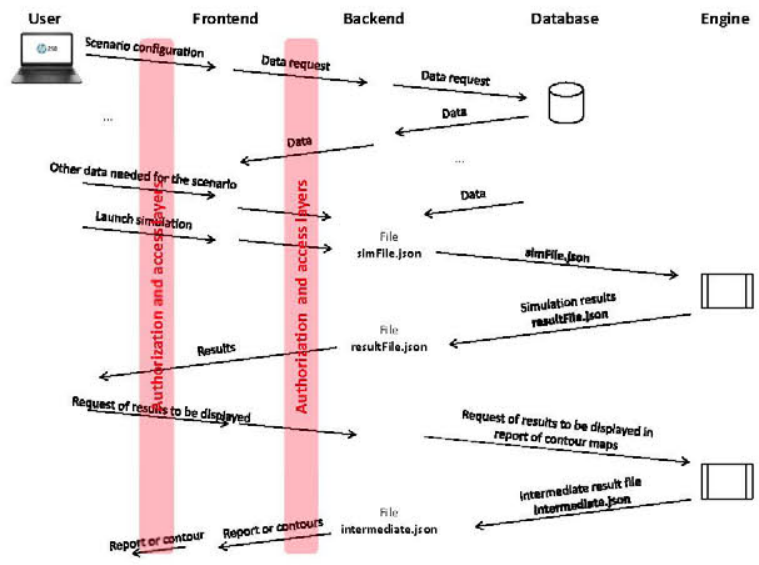

Fig. 3. Flowchart of the simulation process in Hispasim.

\section{CASE STUdies}

The case study scenario is defined to provide a DTH service to Brazil, which corresponds to a forward scenario in HispaSim. User defines the service (30 Mbps), the hub located in Brazil, and remotes are equipped with a small terminal. Modem technology is DVB-S2 and the QPSK 8/9 MODCOD is selected (CCM mode).

Fig. 4 shows the contour plots of availability over $99 \%$ of the DTH scenario. Simulation results show a decay in the availability in the area near Amazonia due to the increased rain attenuation experienced there. Other scenarios allow the computation of the required antenna (diameter, $\mathrm{G} / \mathrm{T}$ ) to provide the DTH service.

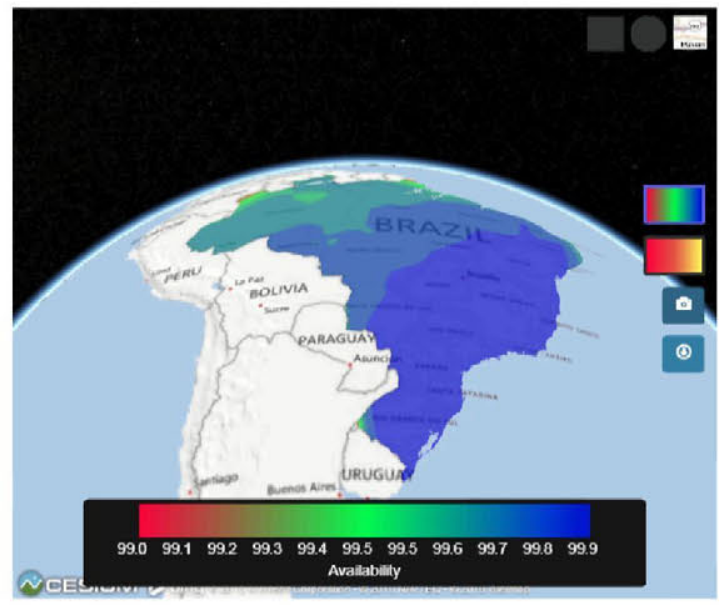

Fig. 4. Availability in the case study of a DTH scenario (3D map).
The user can download the image file of the results or generate a KML file to export the result contours to e.g. Google Earth. User can also provide a template that includes the image file in a particular location in the report.

\section{CONCLUSIONS AND EVOLUTION}

A novel web based application (HispaSim) for the analysis and optimization of satellite link budget has been presented. The application also permits the administration of users and database management, along with report generation using userdefined templates. Modem technologies defined as DVBS2/DVB-S2x are considered in the first version with different EIRP modes and MODCOD operation. Results are displayed in the form of contour maps using a selectable map data provider and reports (summary, tables, transmission plans).

HispaSim web application has been designed based on the requirements provided by Hispasat satellite operator in collaboration with GMV. Thus, it is used in practice to analyze practical satellite communications solutions required by diverse customers.

Evolution of HispaSim presents several opportunities, from new functionalities (e.g. new modem technologies) to development of a version for mobile devices and tablets, or the use of a distributed calculation platform. Embedding the server and frontend into a native hosted application is also possible by means of different technologies, which could allow us to create an offline version.

Also, the chosen technologies and architecture offer a great deal of flexibility on its deployment and integration with other platforms, and its modular nature allows us to keep improving the different components and even adding new ones if required while maintaining backwards compatibility.

\section{ACKNOWLEDGMENT}

Authors would like to thank GMV and Hispasat for the support of this project. Authors would also like to acknowledge the Spanish Government, Ministry of Economy, National Program of Research, Development and Innovation for the support of the project ENABLING-5G "ENABLING INNOVATIVE RADIO TECHNOLOGIES FOR 5G NETWORKS" (project number TEC2014-55735-C3-1-R).

\section{REFERENCES}

[1] ETSI, Digital Video Broadcasting (DVB); Second generation framing structure, channel coding and modulation systems for Broadcasting, Interactive Services, News Gathering and other broadband satellite applications. ETSI EN 302307 v1.2.1, 2009.

[2] ETSI, Digital Video Broadcasting (DVB); Second generation framing structure, channel coding and modulation systems for Broadcasting, Interactive Services, News Gathering and other broadband satellite applications; Part 2: DVB-S2 Extensions (DVB-S2X), (2014-04). ETSI EN 302 307-2 v1.1.1, 2014.

[3] Cesium, https://cesiumjs.org/.

[4] JSON format, http://www.json.org/. 